\title{
Note: A pairwise form of the Ewald sum for non-neutral systems
}

\author{
Shasha Yi, ${ }^{1}$ Cong Pan, ${ }^{1}$ and Zhonghan $\mathrm{Hu}^{1,}, *$ \\ ${ }^{1}$ State Key Laboratory of Supramolecular Structure and Materials, \\ College of Chemistry, Jilin University, Changchun, 130012, P. R. China
}

(Dated: April 17, 2018)

\begin{abstract}
Using an example of a mixed discrete-continuum representation of charges under the periodic boundary condition, we show that the exact pairwise form of the Ewald sum, which is well-defined even if the system is non-neutral, provides a natural starting point for deriving unambiguous Coulomb energies that must remove all spurious dependence on the choice of the Ewald screening factor.
\end{abstract}

In a recent article we derived a pairwise formulation for the Ewald sum associated with any inifinte boundary term[4]. This formulation has an intuitive interpretation of the contribution from the background charge that results in well-defined electrostatic energies. One of the main advantages of this formulation is that, as opposed to other proposed derivations of the Ewald-type algorithm for non-neutral systems (e.g. [18]), one can remove all spurious dependence of the energy on the Ewald screening factor.

Let us consider a system of $N$ discrete point charges $\left(q_{j}, \mathbf{r}_{j}\right)$ with $j=1,2, \cdots, N$ in a cuboid unit cell specified by $L_{x}, L_{y}, L_{z}$ and their infinite periodic images $\left(q_{j}, \mathbf{r}_{j}+\mathbf{n}\right)$ where $\mathbf{n}$ stands for $\left(n_{x} L_{x}, n_{y} L_{y}, n_{z} L_{z}\right)$ with $n_{x}, n_{y}$, and $n_{z}$ integers. The usual Ewald3D sum under the tinfoil boundary condition (e3dtf) for the Coulomb energy of the unit cell reads $1-3$ ]

$$
\begin{aligned}
\mathcal{U}^{\mathrm{e} 3 \mathrm{dtf}} & =\frac{1}{2} \sum_{i, j=1}^{N} q_{i} q_{j} \sum_{\mathbf{n}} \frac{\operatorname{erfc}\left(\alpha\left|\mathbf{r}_{i j}+\mathbf{n}\right|\right)}{\left|\mathbf{r}_{i j}+\mathbf{n}\right|} \\
& -\frac{\alpha}{\sqrt{\pi}} \sum_{j=1}^{N} q_{j}^{2}+\frac{2 \pi}{V} \sum_{\mathbf{k} \neq \mathbf{0}} \frac{e^{-k^{2} /\left(4 \alpha^{2}\right)}}{k^{2}}\left|\sum_{j=1}^{N} q_{j} e^{i \mathbf{k} \cdot \mathbf{r}_{j}}\right|^{2},
\end{aligned}
$$

where $\mathbf{r}_{i j}=\mathbf{r}_{i}-\mathbf{r}_{j}, V=L_{x} L_{y} L_{z}$ and $\mathbf{k}=$ $2 \pi\left(k_{x} / L_{x}, k_{y} / L_{y}, k_{z} / L_{z}\right)$ with $k_{x}, k_{y}$, and $k_{z}$ integers. The prime indicates that the $i=j$ terms are omitted when $\mathbf{n}=0$. The parameter $\alpha \in(0, \infty)$ is a screening factor that determines the relative proportion of the real and reciprocal space sums. However, $\mathcal{U}^{\text {e3dtf }}$ uniformly and absolutely converges to an $\alpha$-independent value for any given non-overlapping configuration. Under the electroneutrality condition, $\sum_{j=1}^{N} q_{j}=0$, $\mathcal{U}^{\text {e3dtf }}$ can be exactly re-expressed as a conventional pairwise form (see Fig. 1 and eqs. (28)-(34) of ref.44])

$$
\mathcal{U}^{\mathrm{e} 3 \mathrm{dtf}}=\sum_{i<j}^{N} q_{i} q_{j} \nu^{\mathrm{e} 3 \mathrm{dtf}}\left(\mathbf{r}_{i}-\mathbf{r}_{j}\right)
$$

where

$$
\begin{aligned}
\nu^{\mathrm{e} 3 \mathrm{dtf}}(\mathbf{r})=\tau^{3 \mathrm{D}}+ & \sum_{\mathbf{n}} \frac{\operatorname{erfc}(\alpha|\mathbf{r}+\mathbf{n}|)}{|\mathbf{r}+\mathbf{n}|}+ \\
& \frac{4 \pi}{V} \sum_{\mathbf{k} \neq \mathbf{0}} \frac{e^{-k^{2} /\left(4 \alpha^{2}\right)} e^{i \mathbf{k} \cdot \mathbf{r}}}{k^{2}}-\frac{\pi}{\alpha^{2} V} .
\end{aligned}
$$

The constant $\tau^{3 \mathrm{D}}$ independent of $\mathbf{r}$ and $\alpha$ is given by

$$
\begin{array}{r}
\tau^{3 \mathrm{D}}=\frac{\pi}{\alpha^{2} V}+\frac{2 \alpha}{\sqrt{\pi}}-\sum_{\mathbf{n} \neq \mathbf{0}} \frac{\operatorname{erfc}(\alpha|\mathbf{n}|)}{|\mathbf{n}|} \\
-\frac{4 \pi}{V} \sum_{\mathbf{k} \neq \mathbf{0}} \frac{e^{-k^{2} /\left(4 \alpha^{2}\right)}}{k^{2}}
\end{array}
$$

Both $\tau^{3 \mathrm{D}}$ and $\nu^{\mathrm{e} 3 \mathrm{dtf}}(\mathbf{r})$ absolutely and uniformly converge to $\alpha$-independent values for any $\alpha \in(0, \infty)$. Taking $\alpha \rightarrow \infty$ in eq. (3), a more concise form for $\nu^{\mathrm{e} 3 \mathrm{dtf}}(\mathbf{r})$ formally reads

$$
\nu^{\mathrm{e} 3 \mathrm{dtf}}(\mathbf{r})=\tau^{3 \mathrm{D}}+\frac{4 \pi}{V} \sum_{\mathbf{k} \neq \mathbf{0}} \frac{e^{i \mathbf{k} \cdot \mathbf{r}}}{k^{2}} .
$$

When the system is non-neutral, $\sum_{j=1}^{N} q_{j} \neq 0, \mathcal{U}^{\text {e3dtf }}$ of eq.(1) still converges but its value depends on $\alpha$. In contrast, the pairwise expression eq. (2) remains well-defined and independent of $\alpha$ because the effect of the background charges has been taken into account by $\nu^{\mathrm{e} 3 \mathrm{dtf}}$. As will be shown below, $\nu^{\mathrm{e} 3 \mathrm{dtf}}$ offers additional convenience when deriving any Ewald sum formula for a continuous distribution of charges.

Rigorous derivations of the Ewald sum[5-7] have shown that the Coulomb energy of the unit cell inside an infinite periodic lattice has an extra shape-dependent term that depends on the asymptotic behavior that the lattice approaches the infinite. Alternatively, this infinite boundary term can be obtained transparently by an analysis of $\mathbf{k} \rightarrow 0$ behavior of the reciprocal space term [4]

$$
\mathcal{U}^{\mathrm{e} 3 \mathrm{~d}}=\mathcal{U}^{\mathrm{e} 3 \mathrm{dtf}}-\frac{\pi}{V} \sum_{i, j}^{N} q_{i} q_{j} \lim _{\mathbf{k} \rightarrow \mathbf{0}} \frac{\left(\mathbf{k} \cdot \mathbf{r}_{i j}\right)^{2}}{k^{2}}
$$

For example, regarding $\lim _{\mathbf{k} \rightarrow 0}$ as $\lim _{k_{z} \rightarrow 0}\left[\lim _{k_{x}, k_{y} \rightarrow 0}\right]$ yields the Ewald sum with the planar infinite boundary term 4$]$

$$
\mathcal{U}_{\mathrm{p}}^{\mathrm{e} 3 \mathrm{~d}}=\mathcal{U}^{\mathrm{e} 3 \mathrm{dtf}}-\frac{\pi}{V} \sum_{i, j}^{N} q_{i} q_{j} z_{i j}^{2}=\sum_{i<j}^{N} q_{i} q_{j} \nu_{\mathrm{p}}^{\mathrm{e} 3 \mathrm{~d}}\left(\mathbf{r}_{i j}\right)
$$


where the corresponding pairwise potential is given by

$$
\nu_{\mathrm{p}}^{\mathrm{e} 3 \mathrm{~d}}(\mathbf{r})=\nu^{\mathrm{e} 3 \mathrm{dtf}}(\mathbf{r})-\frac{2 \pi}{V} z^{2} .
$$

This planar infinite boundary term was actually known since 1980s[5] and was later widely used as a correction to the usual Ewald3D sum with the tinfoil boundary term when simulating planar interfaces [8, 9]. Other efficient and accurate methods using mean-field ideas or the 2D periodic Ewald sum for such systems have been recently developed[10-14]]. Relations among them have been discussed[15, 16]. Moreover, it has been clarified that $\mathcal{U}_{\mathrm{p}}^{\mathrm{e} 3 \mathrm{~d}}$ is in fact an accurate mean-field approximation to the $2 \mathrm{D}$ periodic Ewald sum[17].

In a recent example studied by Levin and coworkers [18], an efficient algorithm was developed to simulate a system of $N_{m}$ mobile ions $\left(q_{j}, \mathbf{r}_{j}\right)$ confined between two charged planar walls by treating the $N-N_{m}$ fixed charges, $\left(q_{s}, \mathbf{r}_{s}\right)$ on the wall as continuum to reduce the computational cost. Neither the fixed charges nor the mobile ions necessarily satisfy the electroneutrality condition. We now use the above pairwise potential $\nu_{\mathrm{p}}^{\mathrm{e}} \mathrm{d}(\mathbf{r})$ of eq. (8) as a starting point to simply and transparently derive the Ewald sum energy that removes a spurious dependence on the screen factor $\alpha$ which appeared in Ref.[18].

Using the Ewald sum with the planar infinite boundary term, the total coulomb energy of the mobile ions can be written as a sum of the mobile-mobile and mobile-fixed components

$$
\mathcal{U}_{\mathrm{p}}^{\mathrm{e} 3 \mathrm{~d}}=\sum_{i<j}^{N_{m}} q_{i} q_{j} \nu_{\mathrm{p}}^{\mathrm{e} 3 \mathrm{~d}}\left(\mathbf{r}_{i j}\right)+\sum_{j=1}^{N_{m}} q_{j} \phi_{\mathrm{mf}}\left(\mathbf{r}_{j}\right),
$$

where each mobile charge $\left(q_{j}, \mathbf{r}_{j}\right)$ interacts with the wall through the potential

$$
\phi_{\mathrm{mf}}(\mathbf{r})=\sum_{s=N_{m}+1}^{N} q_{s} \nu_{\mathrm{p}}^{\mathrm{e} 3 \mathrm{~d}}\left(\mathbf{r}-\mathbf{r}_{s}\right)
$$

As suggested by Levin and coworkers, the discrete fixed charges on the wall $\left(q_{s}, \mathbf{r}_{s}\right)$ might be replaced by a surface charge density distribution $\rho_{s}^{q}(\mathbf{r})=\sigma_{1} \delta\left(z-z_{L}\right)+\sigma_{2} \delta\left(z-z_{R}\right)$. Consequently, one then approximates $\phi_{\mathrm{mf}}(\mathbf{r})$ as

$\phi_{\mathrm{mf}}(\mathbf{r}) \simeq \int_{V} d \mathbf{r}^{\prime} \nu_{\mathrm{p}}^{\mathrm{e} 3 \mathrm{~d}}\left(\mathbf{r}-\mathbf{r}^{\prime}\right) \rho_{s}^{q}\left(\mathbf{r}^{\prime}\right)=2 \pi\left(\sigma_{2}-\sigma_{1}\right) z+C_{1}$ where $C_{1}$, a constant independent of $\mathbf{r}$ is given by

$$
C_{1}=\left(\sigma_{1}+\sigma_{2}\right)\left(L_{x} L_{y} \tau^{3 \mathrm{D}}+\frac{2 \pi L_{z}}{6}\right)+2 \pi\left(\sigma_{1} z_{L}-\sigma_{2} z_{R}\right) .
$$

When integrating $\nu_{\mathrm{p}}^{\mathrm{e} d \mathrm{~d}}$ of eq. (5) and (8), terms in eq. (5) with $k_{x} \neq 0$ or $k_{y} \neq 0$ all vanishes. The surviving terms with $k_{x}=k_{y}=0 ; k_{z} \neq 0$ are related to the Fourier series for $|z|-z^{2} / L_{z}$ on an interval $\left[-L_{z}, L_{z}\right]$

$$
|z|-\frac{z^{2}}{L_{z}}=\frac{L_{z}}{6}-\frac{2}{L_{z}} \sum_{k_{z} \neq 0} \frac{e^{i 2 \pi k_{z} z / L_{z}}}{\left(2 \pi k_{z} / L_{z}\right)^{2}} .
$$

On the other hand, the mobile-mobile component can be rewritten in the usual form

$$
\begin{aligned}
& \sum_{i<j}^{N_{m}} q_{i} q_{j} \nu_{\mathrm{p}}^{\mathrm{e} 3 \mathrm{~d}}\left(\mathbf{r}_{i j}\right)= \frac{1}{2} \sum_{i \neq j}^{N_{m}} q_{i} q_{j} \sum_{\mathbf{n}} \frac{\operatorname{erfc}\left(\alpha\left|\mathbf{r}_{i j}+\mathbf{n}\right|\right)}{\left|\mathbf{r}_{i j}+\mathbf{n}\right|} \\
&+\frac{2 \pi}{V} \sum_{\mathbf{k} \neq \mathbf{0}} \frac{e^{-k^{2} /\left(4 \alpha^{2}\right)}}{k^{2}}\left|\sum_{j=1}^{N} q_{j} e^{i \mathbf{k} \cdot \mathbf{r}_{j}}\right|^{2}+ \\
&+\frac{2 \pi}{V}\left[M_{z}^{2}-Q_{t} G_{z}\right]+C_{2}(\alpha)
\end{aligned}
$$

where $M_{z}, Q_{t}$ and $G_{z}$ are defined as the same in the ref.[18]

$$
M_{z}=\sum_{j=1}^{N_{m}} q_{j} z_{j} ; \quad Q_{t}=\sum_{j=1}^{N_{m}} q_{j} ; \quad G_{z}=\sum_{j=1}^{N_{m}} q_{j} z_{j}^{2},
$$

and the $\alpha$-dependent constant is given by

$$
C_{2}(\alpha)=\sum_{i<j}^{N_{m}} q_{i} q_{j}\left(\tau^{3 \mathrm{D}}-\frac{\pi}{\alpha^{2} V}\right)-\frac{2 \pi}{V} \sum_{j=1}^{N_{m}} q_{j}^{2} \sum_{\mathbf{k} \neq 0} \frac{e^{-k^{2} /\left(4 \alpha^{2}\right)}}{k^{2}} .
$$

Clearly, our expressions (14) and (11) differ from the corresponding eqs.(19) and (21) of Ref.[18] by constants $C_{1}$ and $\mathrm{C}_{2}$ respectively. Both constants are useful for validating the approximations to the Coulomb energies. However, it should be noted that molecular dynamics or Monte-Carlo simulation results [18-20] should not depend on these constants because they are cancelled in the forces and relative energies.

We would like to thank Prof. Claudio Margulis for bringing to our attention ref.[18]. This work was supported by the NSFC (grant no. 21522304) and the Program for JLUSTIRT.
[1] P. P. Ewald, Ann. Phys. Leipzig 64, 253 (1921).

[2] S. W. de Leeuw, J. W. Perram, and E. R. Smith, Proc. R. Soc. London, Ser. A Math. Phys. Sci. 373, 27 (1980).

[3] D. Frenkel and B. Smit, Understanding Molecular Simulation: From Algorithms to Applications (Academic Press, Inc., San Diego, 2002), 2nd ed.

[4] Z. Hu, J. Chem. Theory Comput. 10, 5254 (2014).

[5] E. R. Smith, Proc. R. Soc. London, Ser. A Math. Phys. Sci. 375,
475 (1981).

[6] E. R. Smith, J. Chem. Phys. 128, 174104 (2008).

[7] V. Ballenegger, J. Chem. Phys. 140, 161102 (2014).

[8] J. Neugebauer and M. Scheffler, Phys. Rev. B 46, 16067 (1992).

[9] I.-C. Yeh and M. L. Berkowitz, J. Chem. Phys. 111, 3155 (1999).

[10] Z. Hu and J. D. Weeks, Phys. Rev. Lett. 105, 140602 (2010).

[11] D. Lindbo and A.-K. Tornberg, J. Chem. Phys. 136, 164111 
(2012).

[12] Z. Hu, Chem. Commun. 50, 14397 (2014).

[13] C. Pan and Z. Hu, J. Chem. Theory Comput. 10, 534 (2014).

[14] C. Pan and Z. Hu, Sci. China Chem. 58, 1044 (2015).

[15] S. Yi, C. Pan, and Z. Hu, Chin. Phys. B 24, 120201 (2015).

[16] S. Yi, C. Pan, L. Hu, and Z. Hu, Phys. Chem. Chem. Phys. 19, 18514 (2017)

[17] C. Pan, S. Yi, and Z. Hu, Phys. Chem. Chem. Phys. 19, 4861
(2017).

[18] A. P. dos Santos, M. Girotto, and Y. Levin, J. Chem. Phys. 144, 144103 (2016).

[19] T. Colla, M. Girotto, A. P. dos Santos, and Y. Levin, J. Chem. Phys. 145, 094704 (2016).

[20] A. P. dos Santos, M. Girotto, and Y. Levin, J. Phys. Chem. B 120, 10387 (2016). 